\title{
TOWARD ADAPTIVE (COMPUTER-BASED) HOSPITAL CARE SYSTEMS THAT CAN GROW AND IMPROVE AS A FUNCTION OF USER PARTICIPATION
}

\author{
L. UHR
}

Computer Science Department, University of Wisconsin, 1210 West Dayton St., Madison, Wisconsin 53706 (U.S.A.)

\author{
M. KOCHEN \\ Department of Psychiatry, University of Michigan, Ann Arbor, Michigan 48109 (U.S.A.)
}

(Received: 16 February, 1979)

SUMMARY

This paper describes a computer-based system that would allow doctors, patients, nurses, researchers and experts to participate in medical care in ways that will enhance the usefulness of the system, and will allow the system to grow, adapt and improve as a function of this participation.

\section{SOMMAIRE}

On décrit dans cet article un système, utilisant un ordinateur, qui permettrait aux médecins, malades, infirmières, chercheurs et spécialistes de participer aux soins médicaux d'une manière susceptible d'améliorer l'utilité du système, de lui permettre de se développer, de s'adapter et de se perfectionner en fonction de cette participation.

\section{INTRODUCTION}

The aim of this paper is to demonstrate an adaptive system that is based on the participation of all concerned. First, the system's components are described. The most important is a relational data base of information that is capable of growing and changing as a function of new knowledge input (Kochen, 1960, 1965, 1967).

The paper describes a computer-based system that would allow doctors, patients, nurses, researchers and experts to participate in medical care in ways 191

Int. J. Bio-Medical Computing (10) (1979) 191-203

(C) Elsevier/North-Holland Scientific Publishers Ltd. 
that will enhance the usefulness of the system, and will allow the system to grow, adapt and improve as a function of this participation.

First the system's components are described. The most important is a relational data base of information that is capable of growing and changing as a function of new knowledge input (Kochen, 1960, 1965, 1967) to the system by its users. Users must also be able to ask questions of the system, and of course the system must be able to make inferences that lead to informative answers. Next we will examine the ways these components fit together and interact.

But such a system is still beyond our present capabilities and we could not hope to implement it in one step. So a strategy is discussed for embodying it in a mixed human-computer-based system, one that will encourage research and development that will lead to successively fuller and better modules (whether computer or human based). This type of strategy seems of great interest in itself, as a meta-level methodology for conducting scientific research on this kind of extremely complex system.

Finally, we will present a tentative formulation of the first version of such a system that we propose to implement.

\section{AN EXAMPLE OF A RESPONSIVE MEDICAL SYSTEM}

Consider an example of such a system for a hospital and its various aspects.

\subsection{The storing of information}

Two types of information can be stored in the system's data base (relational network memory). First, there is information about the patients in the hospital, their histories, diagnoses, treatments, etc. The second is information about the different disease syndromes that are typically confronted, their symptoms, treatment, course of progress, etiology, etc.

As much of this information as possible will be stored in the absorbed semantic memory network. But the rest (and this may well include the information for all but a few of the disease syndromes, at least for the first few years) will be stored in traditional ways, including notes, charts, books, and the minds of doctors, nurses, and other 'experts.'

\subsection{The access of information}

Users can interrogate this system (including the non-computer stores of information) by either using a computer terminal, and a simple language that is designed to access the computer-stored information, or by going to a human being (probably somebody who is expert both at the system's language, and also at programming and library science), and asking him for information. This human intermediary might then interrogate the computer, or start asking human experts, 
or try to find the answers himself in the various library stores, or do a combination of all three. Similarly, if the user had asked the computer directly, it might well route the request that it couldn't handle to a human-either this intermediary or the appropriate expert. Or it might tell the user that if he is not happy with the answer it gave him he should turn to a human.

\subsection{Teaching people how to use the system}

The system should try to teach the language that the user must use to interrogate it, as much as possible by leading him along in easy steps. But it should also inform the user that he can ask for special teaching-learning programs, or for manuals, books, or classes or tutorial sessions with human teachers.

\subsection{Adding new information to the system}

The system must also encourage all users to add information to the system both in the form of new facts and knowledge, but also new generalisations, and suggestions about new techniques that will improve the system. If possible, language with which the user asks for information should be rich enough for such additions and suggestions (and also for the teaching/learning just discussed). But there may well be a need for a different special-purpose language for each such task, and that in turn will lead to a need for a gamut of ways in which the user is helped and taught to use each language.

\subsection{Evaluating suggested changes to the system}

Any changes that the user suggests for the system must be carefully evaluated before they are actually made. This must take several steps: first a group of people responsible for the system will try to evaluate them. But they should serve only as a filter for wrong information and silly ideas, and to keep the system from being inundated. The real evaluation will be made by adding the new material in an experimental mode, as a new alternative, by broadcasting the existence of this alternative to potentially interested users, and by encouraging them to use it and react. The system will question them about it, and then continually disseminate these reactions, in a cycle that encourages further use until the suggestions have been proved good (in which case they will be incorporated into the more permanent system), or bad (in which case they will be discarded). In this way new facts can be added to existing data bases, say for allergies or heart disease, and also new data bases can be started. Users will even be encouraged to make suggestions, and to implement, such things as new languages, teaching techniques and memory representations.

\subsection{Offering and telling about alternatives}

In fact, the system will be set up so that there are at least 2 alternatives at most of the key points, like the access language or teaching/learning system. Users will 
be queried about which alternative they prefer and find more useful, and why, and thus ongoing evaluation experiments will be made. The people responsible for the system will of course then use this information to help them design new, hopefully improved, alternatives. But they will always be subjected to the same user evaluations and experimental comparisons.

\subsection{An adaptive community}

This means the responsible group must broadcast and disseminate information about various alternatives, encouraging users to try them out, and telling them what other people have felt, all in as fair and objective a way as possible. In a real sense the whole system then becomes an adaptive community, where everybody can play any roles he desires, and the experimental results and evaluations of the whole community guide future development.

Experience along these lines has been accumulated, for example, at the Institute for Living in Hartford, Connecticut, where clinicians, nurses and administrators are learning by interacting with one another and a data base of 'Daily Nursing Notes' how to improve patient treatment. Nurses enter daily observations, encoded into 13 factors such as 'agitation' and 'acceptable behavior,' as well as medication taken, etc. Outputs are displayed graphically as histograms over time. The ability to retrieve records by drugs enabled the hospital's pharmacy to reduce its staff from 2.5 to 1.5 man-years.

The use of terminals in such a hospital has come to be viewed as a status symbol. When a new, anti-computer nursing director came to the Institute for Living, there was a turnover in the nursing staff with a shift toward the use of scientific methods and professionalisation of nursing; nurses began to feel that nurses' aides rather than they should use terminals, while they should move up to historytaking, diagnosis, supervision assessment and care, not documentation. Some nurses and other medical professionals may feel demeaned and dehumanised to have to interact with, 'account' to, a computer after all the years of medical training and experience that they expected to raise them to a higher social status. Disadvantaged members on the hospital staffs and less educated 'para-professionals,' on the other hand, may feel human perhaps for the first time with a computerised system because they are treated like everyone else.

These very important human considerations may well be the primary determinants of success or failure of the kind of system we envisage. The computer, with its potential for status equalisation, may prove to be a catalyst in accelerating the needed adaptations of the hospital community in this respect.

\subsection{The information stored: what the system 'knows'}

Central to the system is a store of information of 'what the system knows.' But this store cannot simply be a library of books, journals, papers and archives. For the information must be stored in some form that can be used. A variety of 
different kinds of 'users' must be able to query the system and access and receive the information that they need.

\section{STORING, RETRIEVING AND ORGANISING KNOWLEDGE}

This is an extremely difficult problem for the computer to handle by itself today. It is the subject of a great deal of active research, in 'information retrieval,' 'data base management,' 'question-answering,' and 'artificial intelligence.' We take the goal to be a 'world encyclopedia' of the sort first suggested by H. G. Wells, and elaborated upon and extended by Kochen $(1972,1975)$. But it is premature to think of storing the Library of Congress plus the British Museum plus our world's entire encyclopedia of knowledge inside a computer system. This is actually fcasiblc in the sense that some storage media have sufficient capacity. But the information would be no more accessible than it presently is: we would still need a large network of technical librarians, and scholars to track down the needed texts. And each user would himself have to do the usual tracking down-thumbing through catalogs, browsing through pages of books and journals, consulting others' bibliographies and, in general playing the role of detective.

Thus such simple storage of material might reduce the size of the Library of Congress to a few feet of black boxes in the computer room. But these would have to be supplemented with individual users' terminals that had convenient graphics devices and techniques for thumbing through pages of materials, and electronically calling for new 'books' and documents. Essentially, the book that weighs several pounds, with 500 pages of 5 " by 8 " print, has been reduced to something less than the size and weight of a speck of dust. This does indeed lead to one very important improvement: a user can access this computer-based library from any terminal anywhere, so long as he can dial the number and pay for the communication charges. These should shortly be very cheap, and access should be quite possible from anywhere. For satellite-based communication systems will give very large and cheap information-carrying capabilities from any point on earth to any other. So a user can call Washington from Madison or Vancouver, or, with his 10 or 20 pound portable $\$ 3000$ terminal (maybe $\$ 1000$ in 5 years) from Ibiza, the South Pole, the Kon Tiki, or Shangri La. Essentially, this means that the user can use cheap, simple, efficient and lazy telesatellite calls to replace a plane trip to Washington to use the Library of Congress, with likely side-trips to New York, Cambridge and/or London, plus requests for books through inter-library loans. Such electronic transfer of information will be orders of magnitude cheaper, in user effort, fuel, time, and convenience.

But the crucial step that is needed is to absorb this information into forms that make them more usable, more accessible, and even more powerful-in the sense that the information is correctly combined and generalised, rather than being 
stored piece-meal, with the hope that human experts will be able to find, and then combine and generalise, and then write an appropriate book that in its turn will be accessible to others who need it. We can expect to make only slow progrcss on this most difficult problem, and what we need is a system that will allow us to incorporate each new step. Thus we will gradually improve the system. Probably even more important, the system will give us a vehicle for evaluating the worth of each particular step, and it seems an especially natural and powerful idea to ask the system's users to participate in this evaluation, and also in the development of new improvements.

Research in question answering systems and semantic memory structures has developed a number of interesting ways to store and represent information in the form of 'list structures' or 'graphs.' The present state of the art can store a particular, relatively small, set of relatively concrete information (e.g., about the weather, geography, parts of the body) as a set of primitive units that are connected by appropriate relations. Some systems combine primitive units into higher-level compounds, and further combine primitives and compounds, and group them into equivalence classes.

\subsection{Offering, comparing and improving upon alternatives}

Probably the most reasonable way to incorporate such systems into the larger system is as follows: (this is an instructive example illustrating important aspects of the system). At least 2 such systems should be made available, with stored information networks that cover the same material. In addition, this material will be stored in the unabsorbed form of ordinary books and documents. The existence of all these systems will be 'broadcast' (see below) to all users, or at least all potentially interested users. For the medical setting this means that such areas as diagnosis and treatment of several types of disease groups-say cancer or allergies-would be handled, and doctors, nurses and other interested parties would be told of the existence of the several alternative systems, along with information about how to use them, and how to learn to use them. (Once again, ultimately the system may do the teaching, but for now we would have a mixture of terminal-based teaching systems, ordinary books and manuals, and human teachers.)

\subsection{Encouraging user participation}

The system will automatically collect data on how much each of these alternative subsystems is used. When and if problems of space arise, those systems that are redundant in the information they handle and use the least will be obvious candidates for discard. But with only a little trouble we can have the system do much better: it can ask a user (probably when he signs off) whether he has found what he wanted, how helpful the system was, how efficient and easy it was to use, how much he prefers it over other alternatives, and, to the extent that the user is willing to answer, a variety of other questions. 
Users should be allowed to ignore or cut short such questions. But they should also be given benefits for answering them - first the likelihood of more useful systems, but also more tangible short-term benefits, e.g., cheaper rates on the computer, more computer time, more human help, etc. In general the system's philosophy is one of trying to get every user to participate-in useful ways - as much as possible; but not by pushing him but rather by involving him, and demonstrating the consequent fruits of this participation.

\subsection{Languages and techniques for communication}

A relatively simple (state-of-the-art) language in which users can query the system and input new information is needed. Today we can implement a somewhat awkward simple stilted English-like language. Probably more typical, and cheaper in terms of computer time needed to analyse it, is a language closer to the format of the system's network (relational) data base, for example in simple functional notation, like NAME (RIVERS IN ARIZONA). The language called MICRO is an example. A typical command is: 'FIND IN JOBS WHERE ZIP IS 48104.' It is very inexpensive and simple to use, maintain, update, and to change the data structure (Kahn et al., 1974).

Once again, at least 2 such languages should be implemented and made available to all users, so that data can be collected, and comparison experiments run, as to their relative usefulness and value.

Each such language should be able to handle all kinds of user interactions with the system. But if that is not feasible then users who want to perform several functions will have to use several different languages. (Again, this is a complex research issue to be solved experimentally - are several relatively simpler languages better, and in what ways and for what users, than a single more general language?)

The user will always have the alternative of not using any of these languages, but rather going to a human being who will help and serve as his interface. Thus a user can choose not to try to learn any language, and not to try to use the system's terminal. Rather, the human he goes to will be somebody who serves these mechanical functions for him. Once again, there should be incentives that draw the user closer to the system, and make it more attractive for him to make the effort to learn and do things himself. These might include his having to pay the additional costs of this human intermediary, or having to wait until such a limited resource becomes available. On the positive side, the languages and the terminals should be made as attractive, as powerful, and as easy to learn as possible.

\section{TEACHING AND LEARNING AND DISSEMINATION OF INFORMATION ABOUT THE USES OF THE SYSTEM}

Ideally, such a system should be usable without any special training. But we will 
not achieve such an ideal until we have truly 'intelligent' systems. To as great an extent as possible, the system should teach the user how to use it, and should demonstrate its values, powers and beauties. This means that the system needs the following sub-systems:

(i) Teaching-learning systems must help the user do whatever set of things he wants to do.

(ii) The user must be made aware of the facilities that the system offers, and their potential values and benefits.

\subsection{Teaching-learning subsystems}

To as great an extent as possible, the system should teach the user through interactions on the console. Further, the user should learn by doing, that is by actually using the system. He should be able to start by doing something very simple, that he is clearly capable of doing before any learning. For example, he might be told to type 'HELLO' or 'HELP' and then receive a response like 'ASK FOR INFORMATION IN THE FOLLOWING WAY: E.G. IF YOU ASKED: "GIVE THE PRIMARY SYMPTOMS OF STRYCHNINE POISONING" THE ANSWER WOULD BE "FLUSHING, PALPITATIONS, ..."' He would gradually be taught simpler formats, options, limits, etc. The system would evaluate his progress at each step, and compose an appropriate next step.

This opens up the whole rich and complex problem of computers in education, and we cannot today expect a finished product. Rather, it is just such a system as this that can serve as an ideal vehicle and test-bed for the development of such teaching/learning systems. Once again, at least 2 alternative systems would be desirable, so that information could be collected and disseminated as to their comparative values and usefulness. And among the alternatives must be 2 additional non-computer-based methods: written manuals and books (including ones that have the user confront and use the terminal as desirable), and also human teachers-both in traditional classes, and as tutors. Once again, gentle pressures and lures might be used to entice the user toward the more advanced automatic techniques, but it seems best to simply charge the user the true costs, and thus encourage him to make his choices within the economics of the world in which he (thinks he) lives at the time he makes these choices. (Here we might legitimately want to present a different set of economic constraints, the ones that we predict will be operative in the future periods for which we are developing the system.)

We thus once again are in a position where the user has a range of choicesfrom the traditional ones that he presently uses to several of the most advanced we are capable of devising. These choices are set within a system that fairly describes, collects information about, and evaluates their comparative valuc-an ideal test-best within which to learn about, improve, and replace these choices. Thus the system itself is designed to help as much as possible to move in directions that will improve it. 


\subsection{Disseminating and broadcasting information}

Such a system must make active attempts to keep users, and prospective users as well informed as possible about several different types of things: (1) Potential users should be told what they might gain by using the system-the kinds of things that it does, and its potential values. (2) The system should explain the alternative ways of using the system, and the alternative ways of learning how to use each aspect of the system. (3) The system should give information about the relative values of the different alternatives that the system makes available. This should include the results of its own experiments and surveys, and also quotes and references about what people and evaluation groups have said about it, and also suggestions as to where to get further information.

Very strict control must be exercised to make this information as truthful, objective and fair as possible. People should be kept informed, and encouraged to try out new possible goodies. But they should not be misled or propagandised. Each alternative should be given equal time, and a fair description. All costs, benefits and assumptions, especially those that are hidden or obscure, should be broadcast with equal force.

These are of course ideals, but we can probably approach them reasonably well by setting up a committee of overseers who are sympathetic to this point of view and these goals, and who represent a broad spectrum of the divergent attitudes toward the different alternatives. That is, some members of this group should be sceptical and reactionary about the possibility of any of the newfangled alternatives being anything but degradations; others should be enthusiastic about progress for the sake of progress; still others should be hardheaded about systems proving themselves out before being chosen, and so on.

Such a system is, basically, the kind of message center that is presently available on time-sharing systems. A daily 'newspaper' says what's new. The system can put messages that it infers are of special interest to a particular user into his 'letters' file, along with a special flash to him that he has been sent such a message. More permanent information about the total system and its parts can be stored in a 'description and specifications' filc. And any or all of these can be stored in the oldfashioned print-on-paper form, for access by traditional non-computer means. Once again, users' choices should be tallied, analysed, summarised and disseminated, and used to make decisions about improving and replacing sub-systems from among these alternatives.

When a user signs on, whether to get information, or be taught how to use the system, or for some other purpose, he will be asked whether he wants information about the available alternatives. If he does he will be given their names, along with a simple overall evaluation (e.g., 'CONSIDERED BEST BY PHYSICIANS, LESS GOOD BY PATIENTS') and description (e.g., 'GIVES FACTUAL ANSWERS IN TECHNICAL LANGUAGE'). He can then ask for more detailed information, that summarises results of experimental comparisons, and comments 
and testimonials given by the evaluation committee, and individual users. At any point he can decide to try a particular system. But then at any point he can choose to change to another system, or to ask for more evaluative information.

And during this whole process the system will be collecting information about what he is doing, and occasionally ask him for evaluations of the system, as appropriate.

\subsection{Improving the system, using the user}

A crucial aspect of such a system lies in the way that it draws the users, researchers, and implementers together, into an intellectual community where everybody is encouraged to help improve the system as well as to use it. It is vital that the user's languages allow him to make suggestions as to how the system's information, and the system itself, might be changed, and improved. He must further be encouraged, even enticed, to do so. Probably the most vital kind of motivation would come from his simply seeing that his ideas and suggestions are being listened to, evaluated fairly, and then used when judged to be improvements. But he might also be paidwhether in praise, money, or power-for these improvements, much as somebody who suggests improvements in a factory's procedure might be paid, either in a lump or in continuing royalties.

The user will always be in a position where he can relatively passively gain from the system-much as we all gain from a public library. But the system can also collect valuable data as to what users do. And we can take advantage of the user's expertise and special knowledge - both as an expert in some area, as the researcher or physician will be an expert about his experimental results or his patient's experiences, and also as a user of the system. We must make it as attractive and desirable as possible for the user to take a variety of more active roles, from simply answering questions about how useful he has found a system he has used, to asking him to try and compare an alternative system, to asking him to input new knowledge to the system, to making new generalisations, to actually suggesting or even implementing new alternative systems. (We see this kind of thing going on today on time-sharing computers where the users are themselves computer scientists. But to get physicians and researchers to play such an active role we will have to offer them unusually good access to the computer, good programming languages, and rewards. For we are asking them to trade some of their valuable time and effort for something that they may well feel is unrelated to what they are doing. Even if they see steady improvement in the total system, and feel that they have played a real role in that improvement, they may still feel that they could better be replaced by computer scientists. So it may well be that the system will have to develop a new breed of physicians, who perceive helping to improve the system as one of their jobs, much as many of today's physicians perceive public health and unpaid hospital service as part of their jobs. On the other hand it might turn out that the new community of scholars that such a system pulls together and en- 
courages will become such an exciting milieu that enough people will be enthusiastic about entering it and playing an active role. And finally, there are a wide variety of other incentives - money, time, power, publicity, medals, praise, and so on-that can be used.)

Probably inevitably there will be even more difficult things that might be done by a user. Only a few users might be expected to actually implement a new, hopefully improved, language for access of information. But in general users should be encouraged to go as far as they want to, and can.

\section{THE SELF-ORGANIZING AND INDIVIDUATING ASPECTS OF SUCH A SYSTEM}

Alternatives should be set up at as many places as possible, within the framework of an on-going set of experiments, where each set of alternatives is compared, and suggestions for further improvements are encouraged, evaluated and implemented. The obvious places for setting up alternatives are in the representation of the stored information, the languages for access, and for building, these networks of information, and the techniques for teaching prospective users how to interact with the system.

An active group of researchers should also be involved in a full-time effort to formulate and implement new alternatives. But they should be very interested in the ideas of the larger group of users, who are experts in the medical fields for which the system is being implemented. In fact this type of relation, of an adapting and improving community of user-scholar-researcher-implementers, where everybody is encouraged to play whatever variety of roles he prefers, may well help to overcome the barriers that we traditionally see in interdisciplinary research where, for example, a programmer and a physician try to develop a medical diagnosis system, or a programmer and an educator try to develop a teaching-learning system. And it may also overcome the traditional problems in getting people to use a new system that is imposed upon them from the outside. For the users are part of the system.

It is important to note that there is no reason to think the search should be for one single better, or best, subsystem for each task. Rather, it is very likely that, for example, one set of users will prefer one language, and a second set another language. Each should be kept if it fills some niche. Then the attempts at improvements and the comparative experiments and evaluations will focus on each niche. Thus the adaptation can search for an optimal diversity, of different subsystems filling different needs, rather than for a stultifying straitjacket of enforced uniformity.

\section{A BRIEF DESCRIPTION OF THE INITIAL IMPLEMENTATION}

Rather than begin with a second representation for information, we will implement 
a translator capability that will allow a user to define a new format and will automatically translate the present data base into that new representation. The user is not really concerned with how data is internally represented and should not be burdened with that. It may, however, make a big difference in not only the efficiency of the system but in its power and effectiveness as well.

The teaching systems will be of 4 types:

(i) A typical branching program will be developed to go through the different features of the user languagc.

(ii) A parsing model will be developed, and this model will generate explanatory examples, questions, hints and correctional material as it interacts with the user who is trying to learn about the system.

(iii) A printed primer and manual will be made available to prospective users.

(iv) Human teachers will be available for periodic courses and individual tutoring and consultation.

The data base will store information about symptoms, diagnosis and treatment of 3 or 4 disease entities, probably including cancer, allergies, and poisons.

The broadcast system will put messages into each user's files daily, and print a daily newspaper (both in the computer's files and in printed paper form). It also will print weekly and monthly summaries that describe the available systems, users' reactions, evaluation results, tentative suggestions for new systems, and plans and progress for implementation. (Most of this will be done by the human board of overseers.) Information about each system (and each of its parts) will be offered to a user when he asks to use that system.

\section{REFERENCES}

DAY, L., Computer Conferencing: An Overview, in Computer communication, N. Macon (Ed.), International Council for Computer Communication, Stockholm, 1974, pp. 53-70.

Kahn, M. A., Rumelhart, D. L. and Boyd, B. L., MICRO Information Retrieval System. Technical Reference Manual, Institute of Labor and Industrial Relations, University of Michigan, Ann Arbor, Michigan, 1974.

KocHEN, M., (Ed.) Information for action. Academic Press, New York, 1975.

KoCHEN, M., Evolution of brainlike social organs, in Information for action, M. KocheN (Ed.), Academic Press, New York, 1975, pp. 1-18.

KocHEN, M., WISE: A World Information Synthesis and Encyclopedia, J. Doc., 28 (1972) pp. 322-343.

KoCHEN, M., Adaptive mechanisms in digital concept-processing, in The growth of knowledge, $M$. KOCHEN (Ed.), Wiley, New York, 1967, pp. 185-204.

KoCHEN, M., An adaptive system for directly recording and retrieving information in simple, formal, English-like sentences, in Some problems in information science, M. KOcHEN (Ed.), Metuchen, Scarecrow Press, N.J., 1965, pp. 11-20.

KoCHEN, M. Cognitive mechanisms, IBM Research Center Report, RAP 16, Yorktown Heights, N.Y., 1960.

KuPPERMAN, R. H. and Goldman, S. C., Toward a viable international system : crisis management and computer conferencing, in Computer communication, N. MACON (Ed.), International Council for Computer Communication, Stockholm, 1974, pp. 71-80.

Linstone, H. A. and Turoff, M., The Delphi method: Techniques and applications. Reading, Massachusetts: Addison-Wesley, 1975. 
MACON, N., Views from ICCC '74, in Computer communication, N. MACON (Ed.), International Council for Computer Communication, Stockholm, 1974, pp. 53-86.

Overhage, C. J. and Harman, J. R., Intrex, M.I.T. Press, Cambridge, Massachusetts, 1965.

OVERHAGE, C. J. and HARMAN, J. R., The on-line intellectual community and the information transfer systems at M.I.T. in 1975, reprinted in The Growth of Knowledge, M. KocHEN (Ed.), Wiley, New York, 1967.

Turoff, M., The state of the art: Computer conferencing, in Computer Communication, N. MaCON (Ed.), International Council for Computer Communication. Stockholm, 1974, pp. 81-86. 